\title{
A Novel Energy-Efficient Hexapod Robot Design using a Rotary Encoder-Embedded Weight-Bearing Wheel
}

\author{
Ismaila Adeniyi Kamil $^{*}$ and Ajibola Samson Oladokun \\ Department of Electrical \& Electronic Engineering, University of Ibadan, Ibadan, Nigeria
}

\begin{abstract}
The direct proportionality that exists between the joint actuator rated torque of conventional hexapod robots and the payload mass makes them unsuitable for applications that require energy efficiency. In this paper, we propose a novel hexapod robot design which involves the incorporation of a rotary encoder-embedded weight-bearing wheel to relax the stringent limitations on the choice of the robot's joint actuator torques and the battery capacity. The results of the prototype implementation showed that our design inherits the merits of easy linear distance measurement via the embedded rotary encoder, low actuator torque and high payload capability from wheeled robots.
\end{abstract}

Keywords: Hexapod robots; Joint actuator; Wheeled robots; Weight-bearing wheel; Rotary encoder; Actuator torque

DOI: $10.7176 / \mathrm{CTI} / 8-06$

\section{Introduction}

Mobile robots possess locomotion mechanisms that enable them to move from one position in space to another. Such locomotion mechanisms could be in form of wheels, as is the case in wheeled robots, or limbs, as found in walking or limbed robots. Locomotion speed is one of the main merits that wheeled robots possess over limbed robots, as the rotation of the rotary actuators of wheeled robots directly correspond to translational motion of the robot chassis (Neal \& Alonzo, 2016), while the rotary actuators of limbed robots require a sequence of rotations to produce translational motion (Travis \& James, 2016). Wheeled robots are also easier to control than limbed robots as the joint actuators of limbed robots require precise angular rotations as opposed to wheeled robot actuators, which could attain multiple revolutions per minute to perform their intended functions. Wheeled robots also hold the advantage of relatively low development cost over limbed robots as wheeled robots require as little as 4 rotary actuators to enable translational motion (Neal \& Alonzo, 2016), while limbed robots could require as much as 18 joint actuators (Konstantinos, et al., 2017). The inherent function of rotary actuators in wheeled and limbed robots also favours wheeled robots. Since wheeled robot actuators only require enough torque to overcome inertia, they can withstand the weight of heavy payload unlike limbed robot actuators whose functions include lifting the entire weight of the robot chassis and the payload off the ground (Wensing, et al., 2017). This difference in actuator function has led to the need for limbed robots to either possess high-torque joint actuators or low-mass chassis and payload.

Though limbed robots are slower in translational locomotion than wheeled robots, they offer greater balance while moving over uneven terrains (Pope, et al., 2016). The physical orientation of wheeled robots while in motion is directly dependent on the shape and form of the terrain on which it moves. Thus, an uneven terrain can cause a wheeled robot to have a tilted orientation while in motion, which could result in the robot toppling-over. Conversely, limbed robots can be configured in such a way that they can isolate their main chassis from terrain irregularities and always retain their horizontal orientation regardless of the shape and form of the terrain on which they move. The inherent ability of limbed robots to shift their centre of mass also gives them an edge over wheeled robots in terms of stability as it enables them to compensate for alterations in their centre of gravity due to uneven terrains (Perrin, et al., 2016).

Furthermore, what limbed robots lack in speed, they make up for it by having a better precision of motion than wheeled robots (Oriol, et al., 2016). The position and orientation of a limbed robot's foothold can be predetermined with accuracy relative to the base frame of the robot, via inverse kinematic algorithms, such that the robot has complete control over where it places its limb end-effectors, which in turn gives it the ability to avoid undesirable footholds. This gives limbed robots the ability to make sharp turns and even turn on a spot without making translational motion (Travis \& James, 2016). It also enables them to cover precise linear distances unlike wheeled robots that could easily overshoot a desired covered distance (Pope, et al., 2016). 
Wheeled robots lack the flexibility of control in the position and orientation of their footholds, making it more difficult for them to make sharp turns or turn about a point without having to making series of translational movements (Neal \& Alonzo, 2016).

The locomotion technique of limbed robots could either be static or dynamic. Limbed robots with static locomotion mechanisms have centre of gravity that are fairly fixed while in motion as they only shift by small angles while the robot limbs move (Oriol, et al., 2016). Conversely, limbed robots with dynamic motion technique utilize gravity and inertia to aid their translational motion, thus having varying patterns of motion (Konstantinos, et al., 2017). Unlike in static limbed robots, the centre of gravity of dynamic robots shift by great angles as the robot tries to achieve balance while in motion (Travis \& James, 2016). This is due to the fact that dynamic robots are inherently not stable while in motion due to their mechanical configuration, creating the risk of the robot toppling-over (Pope, et al., 2016). This necessitates the need for dynamic robots to shift their centre of gravity in the opposite direction every time they lean to one direction. This results in a complex control system for dynamic limbed robots as opposed to static robots, as their control system do not possess a dedicated control mechanism for centre of gravity alteration (Perrin, et al., 2016).

Walking robots are developed to have from two legs (Travis \& James, 2016) to as many as a hundred legs for translational locomotion (Konstantinos, et al., 2017). Bipedal robots, which are walking robots with two legs in a similar manner as humans, are statically inherently unstable as they tend to lean in one direction even while being stationary. This necessitates the need for them to utilize dynamic control mechanisms to shift the robot's centre of gravity in compensation (Travis \& James, 2016). Four-legged robots have an advantage over bipedal robots in terms of stability as they are statically stable while stationary. But when one leg is raised, the other three legs become insufficient to aid the robot to maintain its stability. This necessitates the need for the centre of gravity to be shifted such that the weight of the robot's chassis rests on the three legs. Thus, like the bipedal robot, four-legged robots also require dynamic control (Wensing, et al., 2017). The walking robots with the least number of legs capable of utilizing static control are six-legged robots, also called hexapod robots. This is because hexapod robots are capable of maintaining static stability even when three legs are off the ground. This results in a simple control mechanism for hexapod robots and makes them the easiest walking robot to develop (Oriol, et al., 2016). Limbed robots with more than six legs utilize static control but require more power and more development costs as they possess more joint actuators and links. Hexapod robots could either utilize a tripod gait like insects do, or a wave gait which does not exist in nature (Oriol, et al., 2016). Regardless of the walking gait used by a hexapod, the requirement for its joint actuators to possess sufficient torque to lift its main chassis mass makes wheeled robots the preferred choice in most robotic applications.

\section{Related Works}

Due to preference for wheeled robots in most applications, conventional hexapod robots are usually developed for entertainment purposes, as is the case with hexapod robot toys. The Hexbug project, a brand of automated toys developed by the Innovation First toy company, include small hexapods that react to sounds and pressure on their antennae, and can be controlled wirelessly. It also includes small hexapods that react to obstacles by rapidly changing direction and return to their feet when they fall on their backs. Hexapods are also developed for competitive purposes between students of robotic engineering in universities. This serves as a creative avenue for the students to hone their skills in control systems design. Hexapod robots are also developed for biological purposes to test biological theories about insect locomotion, motor control, and neurobiology.

Most hexapod robots are developed using conventional hexapod designs, but there are unique hexapod robot designs with special features for unique applications (Bailey, et al., 1999), one of which is the Robots in Scansorial Environments (RiSE) climbing robot by Boston Dynamics. The RiSE hexapod robot was developed to climb walls, trees, and other vertical terrains with the aid of micro-claw feet to clamp-on to textured surfaces (Spenko, et al., 2006). The RiSE climbing robot was modelled after a 5-cm long climbing insect (Goldman, et al., 2006), as shown Figure 1(a), as the insect's limbs and joints are as modelled in Figure 1(c) to generate a robot model as shown in Figure 1(d). It had the ability to alter its shape to conform to the curvature of vertical platforms and sustained a balanced orientation while performing its climbing function with the aid of a weightshifting tail, as shown in Figure 1(b), which implies that the robot utilizes a dynamic locomotion control system (Asbeck, et al., 2005). The RiSE was developed to have lateral symmetry and tripod walking gait, which are features adopted from walking insects, as shown in Figure 2(a). Figure 2(a-f) show the frontal and sagittal plane simulations, the foot contact forces, the viscous damping in the limbs and joints, the foot trajectories, and the corresponding wall reaction forces (Autumn, et al., 2006). 

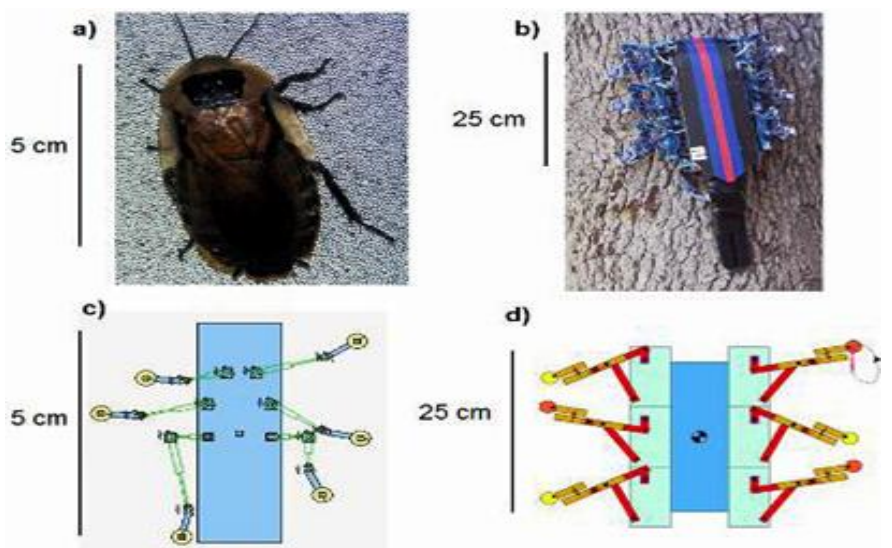

Figure 1. Modelling of RiSE robot (a) Climbing Insect, (b) Robot, (c) Insect Model and (d) Robot Model (Autumn, et al., 2005)

What makes the RiSE robot unique amongst other hexapod design is how specific its application is. It is not practically feasible to develop a wheeled robot for climbing vertical terrain; thus, wall climbing is the only application reserved for limbed robots. In every other robotic application wheeled robot have mostly been the robot of choice due to their greater payload to consumed power ratio, but this limitation is not considered in climbing applications. Thus, this makes the RiSE robot one of the most useful and unique hexapod designs today.

Another unique hexapod design worthy of note is the All-Terrain Hex-Limbed Extra-Terrestrial Explorer (ATHLETE) hexapod rover. The ATHLETE hexapod robot was a lunar rover developed by JPL (Jet Propulsion Laboratory) under the guidance of National Aeronautics and Space Administration (NASA). The first prototypes were developed in 2005 and the second-generation prototypes were completed in 2009 and one of these is still operational today. It was developed for the exploration of the moon's surface, to carry cargo and to provide mobility for astronauts. It served as a mobile lunar base for astronauts, giving them the ability to move their heavy equipment closer to lunar sites of interest. The most unique feature that distinguishes it from every other hexapod developed till date is its wheel-on-limb design concept. This concept involved the incorporation of wheels as the end-effectors of the robot's legs. The use of wheels for translational locomotion, which was adopted from wheeled robots, enabled it to travel at high speeds that are not usually associated with walking robots.

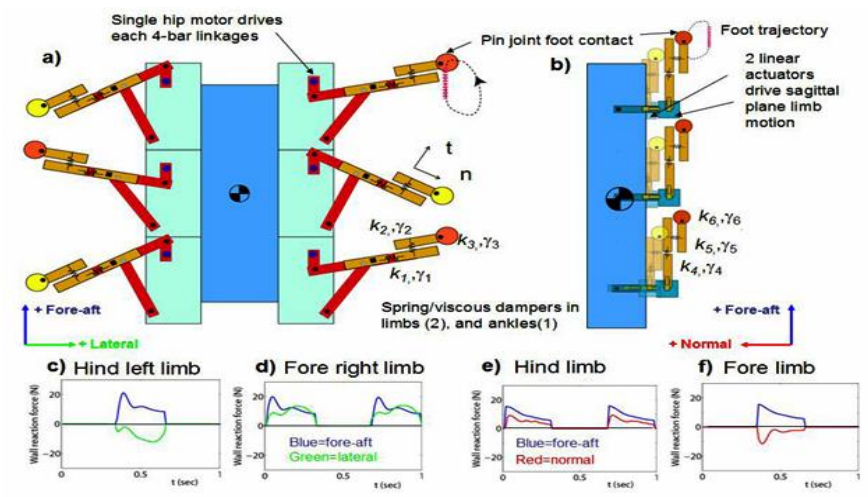

Figure 2. Two RiSE robot simulations in the a) frontal and b) sagittal planes with corresponding wall reaction forces (c-f) (Autumn, et al., 2006)

When the end-effector wheels of the robot struggled on uneven, steep, and extremely soft lunar terrains, the wheels were locked in place by hydraulic power brakes to transform the wheels to feet with which it was able to walk across extreme lunar terrains like a conventional hexapod robot would. Another unique feature of the ATHLETE hexapod that cannot be found in any other hexapod design till date is its ability to split into two independent tripod (three-limbed) robots called tri-ATHLETEs. The ATHLETE hexapod disassembles itself into tri-ATHLETEs to investigate two lunar sites simultaneously and these travel at high speeds like wheeled robots 
on even lunar terrains with firm soil, and resort to walking with their wheels locked when moving over uneven terrains with loose soil (Matt, et al., 2010).

Another unique feature of the ATHLETE hexapod apart from its wheel-on-limb or tri-ATHLETE concepts is its ability to interact with lunar objects by lifting two non-adjacent limbs while shifting its entire body weight to the other four limbs. The wheels of the robot were designed to include wheel hubs that make it possible to attach other tools as the end-effectors of the limbs. With this mechanism, interchangeable tools, such as grippers or augers, could be attached to the two raised limbs to enable the ATHLETE robot to grip lunar objects.

The ATHLETE hexapod robot required more electrical power to move across the lunar surface than an equivalent wheeled robot of the same size. A wheeled robot would only have required power to drive the wheel motors, but the ATHLETE robot powered the wheel motors as well as the joint actuators, which were required to provide sufficient torque to lift the mass of the robot's main chassis of approximately $1000 \mathrm{~kg}$, the mass of the wheels and end-effector tools, and the mass of lunar objects of interest to the astronauts. Though the ATHLETE hexapod utilizes more power than a wheeled robot equivalent, the requirement for locomotion across the unpredictable terrains of the moon's surface necessitated its development regardless of the power implications. The RiSE hexapod robot also suffers from this power implications as its joint actuators were required to provide sufficient torque to lift the weight of the robot chassis and a payload while applying the required force to hold on to the surface of vertical terrains. The hexapod design concept we propose in this paper eliminates these power implications by significantly reducing the torque requirements of the joint actuators (Matt, et al., 2010).

\section{Prototype Hexapod Design}

In order to demonstrate the merits of our hexapod design approach over conventional hexapod designs, we develop a prototype hexapod robot, as shown in Figure 3, to implement our ideas. Each limb of our prototype hexapod robot is composed of 3 degrees of freedom. All the robot's links were machined from $3 \mathrm{~mm}$ rigid Polyvinyl Chloride (PVC) plastic sheets to reduce the chassis weight and ensure that robot can withstand substantial mechanical stress. All the joints of the prototype robot were revolute joints with servo motors serving as the joint actuators. The emphasis of our design approach is not focused on speed; thus, the prototype did not include a wheel-on-limb concept like the ATHLETE robot, which would contribute to increased limb weight. Instead, the focus is on achieving significant reductions in the electrical energy required by the hexapod to execute similar walking sequences and gaits as conventional hexapod robots.

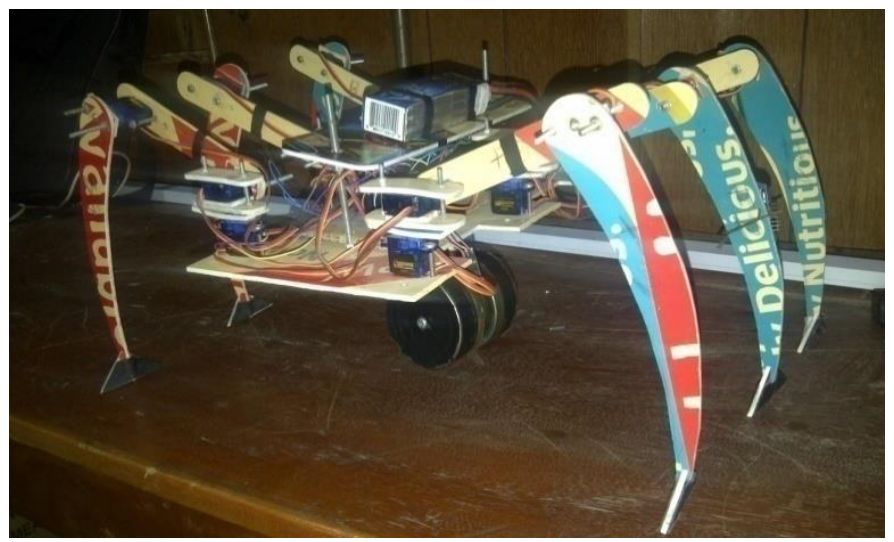

Figure 3. Image of the Prototype Hexapod Robot with the Rotary-Encoded Weight-Bearing Wheel.

\subsection{Link Parameters Derivation}

The developed hexapod robot is basically composed of six identical 3-DoF limbs connected to the main chassis in bi-lateral symmetry, as shown in Figure 4, and Figure 5 shows the derivation of each limb and its frame components. The Denavit-Hartenberg parameters for each limb manipulator are as shown in Table 1, where $a_{i}, \alpha_{i}$, $d_{i}$ and $\theta_{i}$ are the link length, link twist, link offset and joint angles respectively. Equation 1 shows the transformation matrix that defines the position and orientation of the end-effector of each limb relative to its base frame. The inverse kinematic equation, which relates the tip of the limb to the point of connection to the chassis, was obtained from the manipulator frame assignment and the link parameters, as shown in equation 2 . The equation can be solved to obtain the corresponding joint angles, as illustrated in equations 3 to 5 , which are required to move the end-effector of any of the limb manipulators to a specified position and orientation in space 
with respect to their base frames.

Table 1. Derivation of Denavit-Hartenburg Parameters for the Prototype Hexapod Limb Manipulator

\begin{tabular}{|c|c|c|c|c|}
\hline $\begin{array}{l}\text { Link No. } \\
\text { (i ) }\end{array}$ & (m) & $\begin{array}{c}\alpha_{\mathrm{i}} \\
\text { (degree) }\end{array}$ & (m) & $\begin{array}{c}\theta_{\mathrm{i}} \\
\text { (degree) }\end{array}$ \\
\hline 1 & 0.00 & +90 & 0.01 & $\theta_{1}$ \\
\hline 2 & 0.11 & 0 & 0 & $\theta_{2}$ \\
\hline 3 & 0.20 & 0 & 0 & $\theta_{3}$ \\
\hline
\end{tabular}

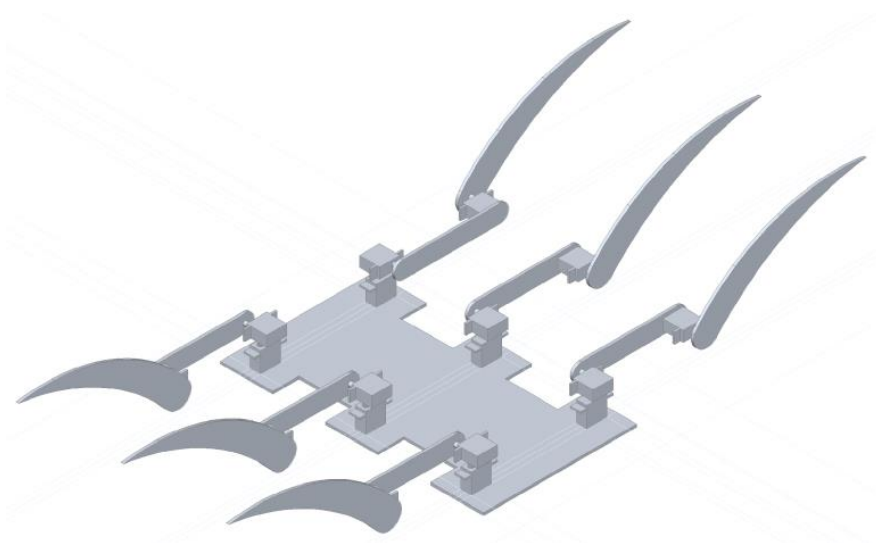

Figure 4. 3D Rendering of Prototype Chassis showing Bi-Lateral Symmetry.

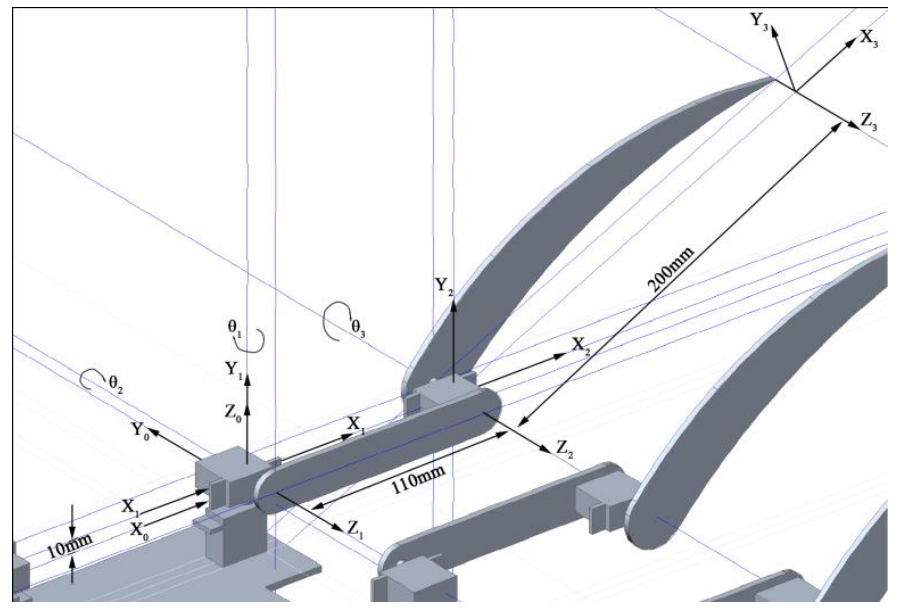

Figure 5. Link Frame Assignment for Hexapod Limb Manipulators. 


$$
\begin{gathered}
{ }_{3}^{0} T=\left[\begin{array}{cccc}
r_{11} & r_{12} & r_{13} & p_{x} \\
r_{21} & r_{22} & r_{23} & p_{y} \\
r_{31} & r_{32} & r_{33} & p_{z} \\
0 & 0 & 0 & 1
\end{array}\right] \\
T={ }_{3}^{0} T={ }_{11}^{0} T_{2}^{1} T_{3}^{2} T \\
\theta_{1}=\tan ^{-1}\left(\frac{-r_{13}}{r_{23}}\right) \\
\theta_{2}=\sin ^{-1}\left(\frac{-0.2 r_{31}+p_{x x}-0.01}{0.11}\right) \\
\theta_{3}=\tan ^{-1}\left(\frac{r_{11} \sin \theta_{1}-r_{21} \cos \theta_{1}}{r_{12} \sin \theta_{1}-r_{22} \cos \theta_{1}}\right)
\end{gathered}
$$

\subsection{Rotary-Encoded Wheel Incorporation}

The unique feature of the developed hexapod robot is the incorporation of a rotary encoder-embedded weightbearing wheel. The functions of the wheel are to simply bear the robot chassis and payload weight, and measure the linear distance covered by the robot. As shown in the 3D rendering image in Figure 6, the wheel is installed along the center of mass of the hexapod robot such that it carries the weight of the robot as well as the weight of potential payloads. The orientation of the wheel is designed such that the robot is free to move in the forward and reverse directions, while its embedded rotary encoder measures the linear distance covered. The placement of the wheel along the robot's center of mass also ensures that the hexapod retains its flexibility of motion and its inherent ability to turn on a spot, a trait that gives it an edge over wheeled robots, as shown in figure 7 . The presence of the wheel does not hinder our hexapod design from executing a tripod walking sequence or a wave walking sequence, as shown by the 3D rendered image in figure 8 . The limbs are free from the responsibility of payload weight bearing and they simply provide sufficient reaction force to tow the robot forward or backwards for linear locomotion. In addition, the wheel is utilized as a fulcrum on which the robot turns about a point in space.

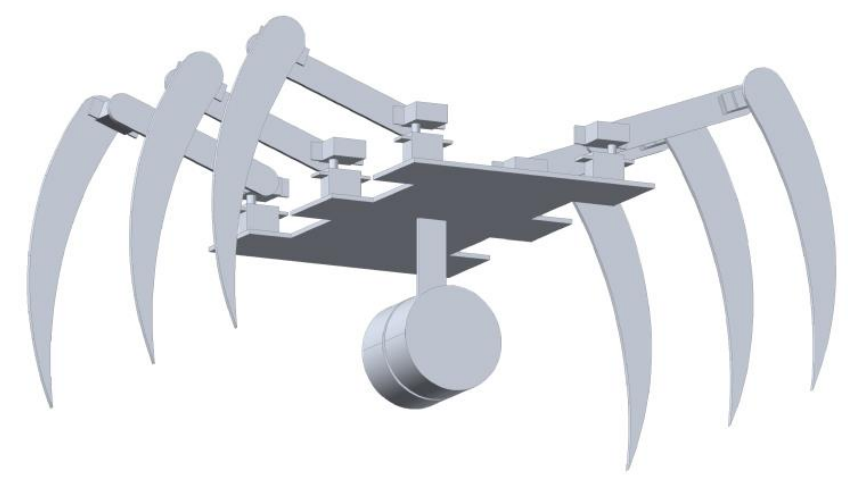

Figure 6. 3D Image of the Developed Hexapod Robot with Centered Weight-Bearing Wheel. 


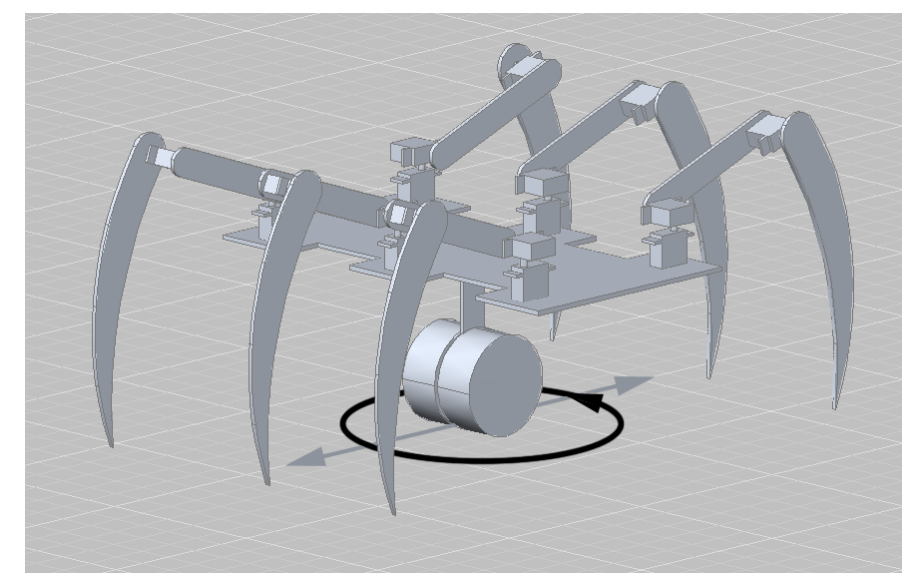

Figure 7. 3D Image of the Prototype Robot with Fixed Wheel Orientation.

The use of a rotary encoder to the measure linear distance covered by the hexapod is a concept adopted from wheeled robots. Conventional hexapod robots have no provision to incorporate the use of rotary encoders and so the robots have to rely on Global Positioning System (GPS) tracking, which is not suitable for measurement of short distances, or image processing, which introduces increased complexity and significant cost to hexapod designs. Rotary encoders are significantly lower in cost and complexity, as opposed to GPS or image processing, and they give consistent and accurate readings as the wheel has a low probability of free-spinning or skidding due to its lack of rotary actuation. Figure 9 shows the 3D rendering of a conventional hexapod design without the wheel.

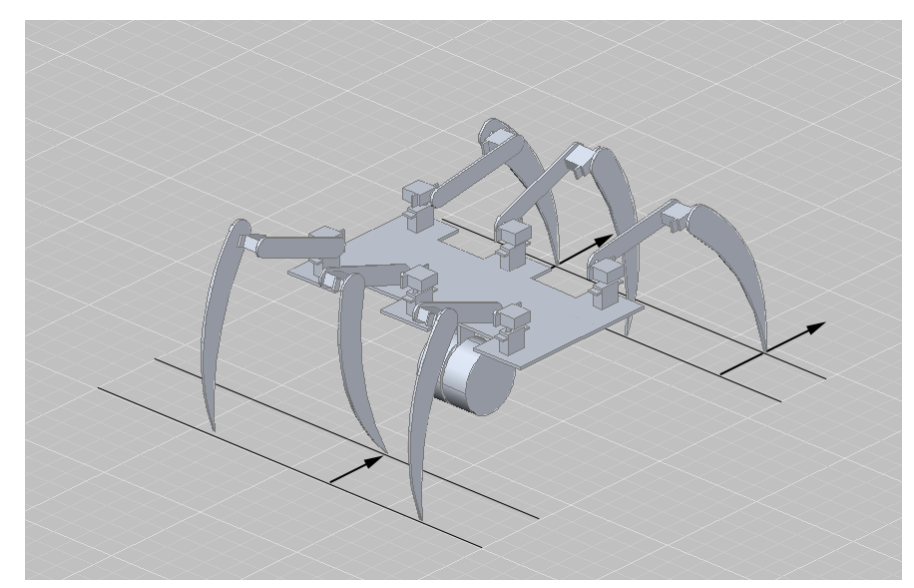

Figure 8. 3D-Rendering of the Prototype Hexapod Executing a Tripod Gait

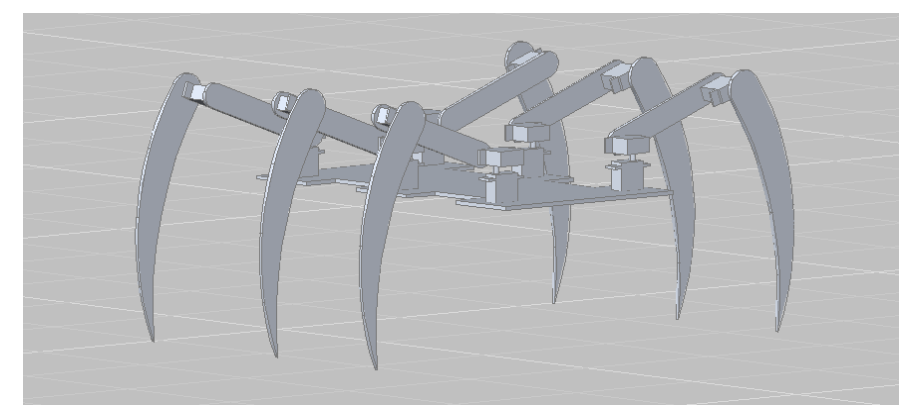

Figure 9. 3D-Rendering of the Prototype Hexapod without the Weight-Bearing Wheel. 


\subsection{Joint Actuator Considerations for the Novel Hexapod Design}

The main merit of the proposed hexapod robot design is that it makes use of joint actuators with significantly low torque, current, power, mass and cost. To verify this claim, the joints of the prototype hexapod robot are controlled by micro servomotors, which are the commercial off-the-shelf (COTS) servomotors with significantly low output torque, current, power and cost. Each micro servo has a mass of $9 \mathrm{~g}$, a stall torque of $180 \mathrm{mN}-\mathrm{m}$, nominal voltage of $5.2 \mathrm{~V}$, nominal current of $250 \mathrm{~mA}$ and stall current of $650 \mathrm{~mA}$. Though, the joint actuators of the prototype hexapod manipulator limbs do not have to possess sufficient torque to lift the chassis weight, they still have to possess sufficient torque to move their corresponding links and actuators of the manipulators. Figure 10 shows the derivation of the upper and lower link masses of the prototype robot limbs. Figure 11 shows the torque required by each of the three joint actuators in one of the manipulator limbs to move corresponding links and joint actuators while taking into consideration the masses of the links and joint actuators. As shown in Figure 11 , the first actuator in the open-loop kinematic chain requires the highest torque, which is obtained to be $42.688 \mathrm{mN}-\mathrm{m}$, of all the three actuators to move corresponding links and actuators. This means that the minimum torque required by the manipulator limbs of our prototype hexapod robot is $42.688 \mathrm{mN}-\mathrm{m}$. The micro servomotors used as the joint actuators in our prototype have mass of $9 \mathrm{~g}$ and maximum torques of $180 \mathrm{mN}-\mathrm{m}$, thus reaffirming their suitability as the joint actuator of choice.

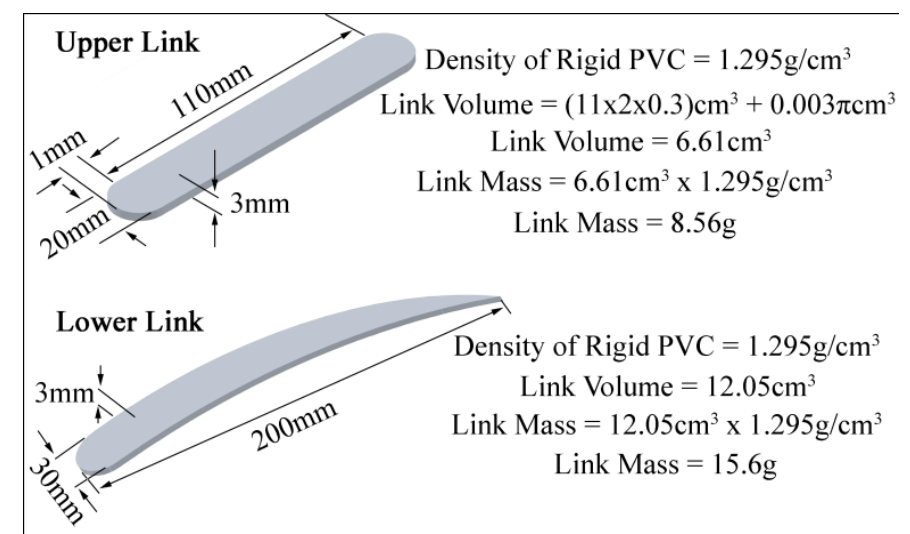

Figure 10. Derivation of Prototype Hexapod Robot Link Masses

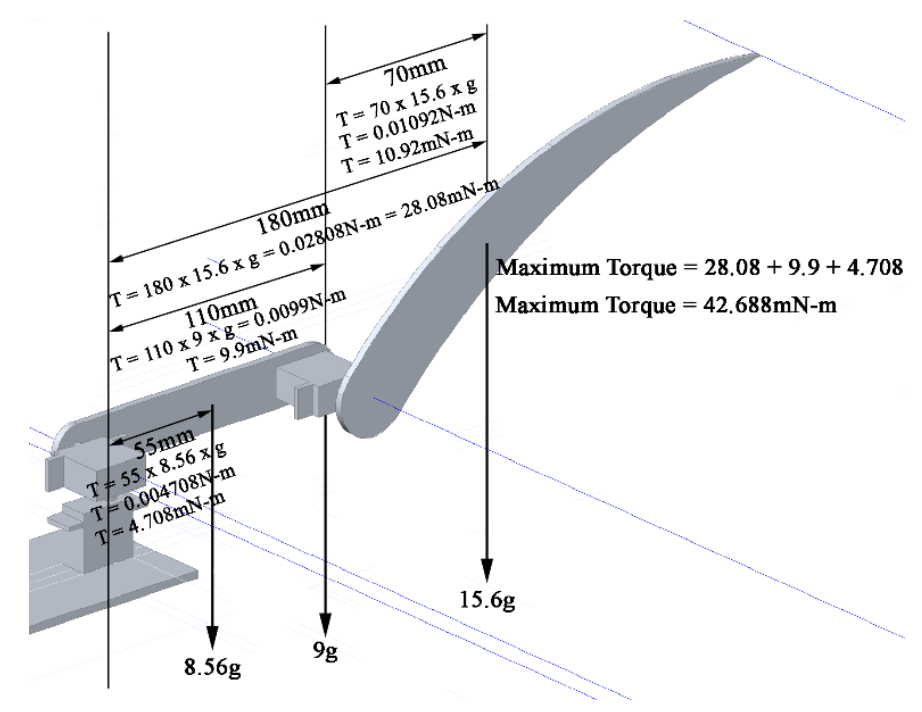

Figure 11. Torque Requirement of the Manipulator Joint Actuators. 


\subsection{Joint Actuator Considerations for Conventional Hexapod Design}

To observe the merit gap between our novel design and the conventional hexapod design, we reassemble our prototype hexapod to have the appearance of conventional hexapods by removing the weight-bearing wheel, as shown in Figure 9. After reverting to the conventional hexapod design, the micro servomotors were incapable of lifting the robot's chassis off the ground as their output torques were insufficient to overcome the weight of the robot and the battery payload. This meant that in order to convert our prototype to a conventional hexapod, it was necessary to upgrade the servomotors to actuators with greater torque. Figure 12 shows that the minimum torque required by our prototype's joint actuators to lift its chassis mass of 590g and battery payload mass of $180 \mathrm{~g}$ is $1520.75 \mathrm{mN}-\mathrm{m}$. To achieve this torque, MG996R metal geared servomotors, which are the COTS servomotor next-in-line to micro servomotors in terms of output torque, were installed as the joint actuators of our prototype hexapod. Its high torque of approximately $2000 \mathrm{mN}-\mathrm{m}$ came at the cost of nominal currents of $900 \mathrm{~mA}$, stall currents of $2.5 \mathrm{~A}$ and maximum power draw of $15 \mathrm{watts}$.

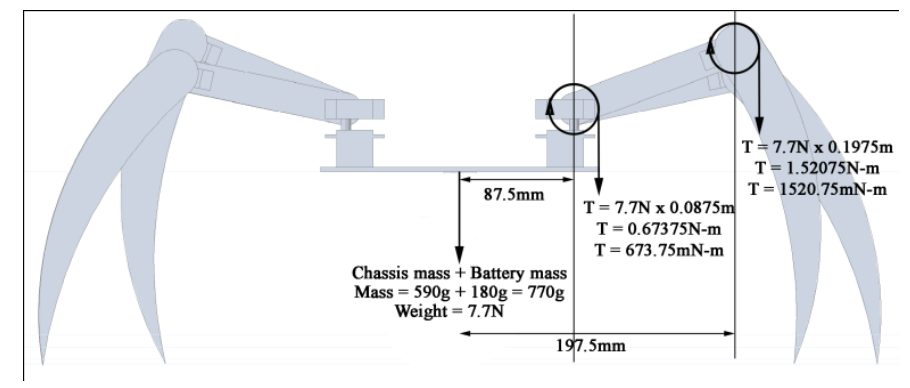

Figure 12. Torque Requirement of the Prototype Robot Joint Actuators without the Weight-Bearing.

\subsection{Stiction and Rolling Resistance Considerations}

In order for the proposed hexapod robot to move in the forward or reverse direction, the wheel has to rotate as the limb manipulators perform tripod or wave gaits. This means that the wheel must overcome every opposing force to its motion. This includes stiction, which is the static friction that tends to prevent stationary surfaces from being set in motion and rolling resistance. Rolling resistance or rolling friction, which is the force resisting the motion of a rotating object when it is in motion, is more apparent when there is deformation at the point of contact between a wheel and a flat surface. This is caused by hysteresis as not all the energy required for deformation of a wheel or horizontal platform is recovered when the pressure is removed. The light weight, high tensile strength and poor elasticity characteristics of tin made it the material of choice for fabricating the wheel of the prototype, as rubber wheels are prone to hysteresis and rolling friction. The stiction and rolling resistance of the hexapod's wheel is negligible on hard surfaces but it is more apparent when the prototype robot traverses across soft terrains as the deformation of the loose terrain introduces hysteresis and significant stiction. The maximum required torque from the micro servomotors, with rated torque of $180 \mathrm{mN}-\mathrm{m}$, to move adjacent links and actuators has been derived to be $42.688 \mathrm{mN}-\mathrm{m}$, thus leaving up to $137 \mathrm{mN}-\mathrm{m}$ of torque, which is assumed to be sufficient to overcome stiction and rolling resistance on reasonably soft terrains.

\section{Results and Discussions}

\subsection{Actuator Voltage Level Analysis}

As earlier stated, the micro servomotors are used as joint actuators of the proposed hexapod robot, and the micro servomotor with the greatest torque demand is the one serving as the joint actuator for frame 1 in all the six manipulator limbs, as it the actuator with the greatest mechanical load. Figure 13 shows the voltage levels, sampled at a $2.4 \mathrm{kHz}$ Analog to Digital Converter (ADC) sampling frequency, of one of the frames 1 micro servomotors while the robot executes tripod walking sequences across a horizontal platform. As shown in this figure, the servomotor is supplied a voltage of $5.2 \mathrm{~V}$ but the applied voltage experiences sharp dips to around $4 \mathrm{~V}$ every time the servomotor attempts to rotate to a new angular position. This voltage drops are due to the high current drawn by the servomotor whenever it starts up. The applied voltage remains constant at $5.2 \mathrm{~V}$ after the servomotor reaches its destination angular position as the motor comes to rest. Similarly, Figure 14 shows the 
voltage levels of a frame 1 joint actuator when the prototype hexapod is reconfigured to look like a conventional hexapod design by removing the wheel and replacing the micro servomotors with the MG996r high torque servomotors. As shown in Figure 14, the high torque servomotor produced more pronounced dips in voltage, with the applied voltage dropping to approximately $1.8 \mathrm{~V}$, which is significantly lower than the voltage dip experienced in the micro servomotor, as it requires more start-up current than that of the micro servomotor.

\subsection{Actuator Current and Power Analysis}

Figure 15 shows the current drawn by a frame 1 micro servomotor, sampled at $2.4 \mathrm{kHz}$ ADC sampling rate, in the proposed prototype hexapod while executing a tripod walking sequence. The micro servomotor is triggered multiple times till its gears achieve the desired angular positioning. This results in multiple current spikes as shown in Figure 15, with the current spikes peaking at approximately 250mA. Obtaining the instantaneous voltage across and current through the micro servomotor at $2.4 \mathrm{kHz}$ ADC sampling rate and evaluating their product yields the plot of the power consumed by the micro servomotor with respect to time, as shown in Figure 17.

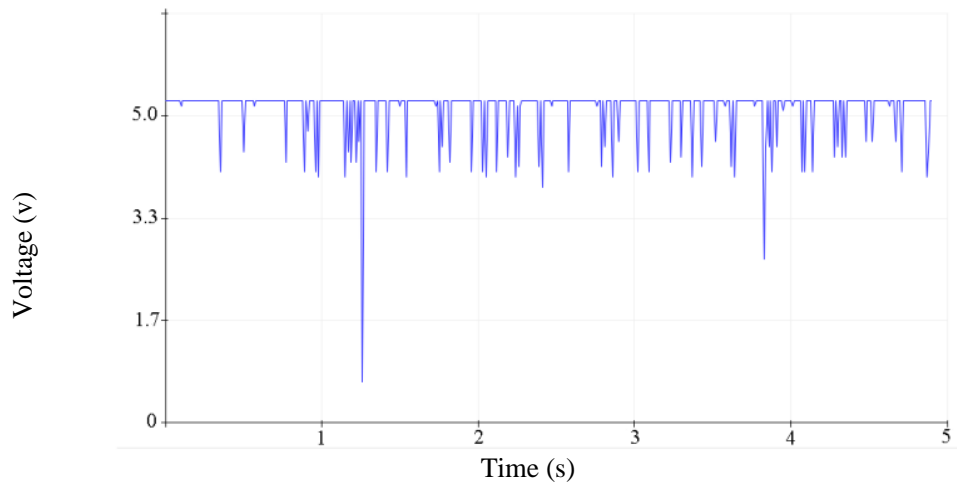

Figure 13. Voltage Levels of Micro Servomotor as Joint Actuator in a Tripod Gait

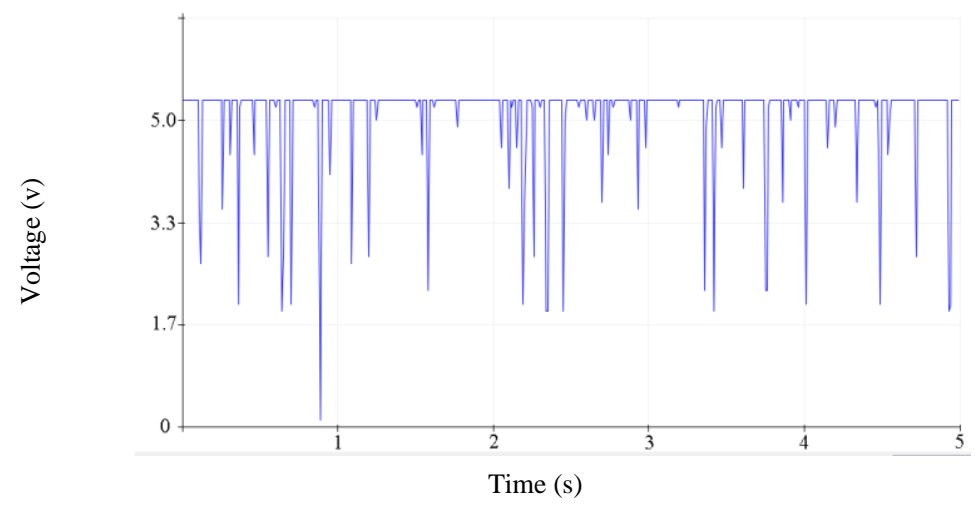

Figure 14. Voltage Levels of MG996r Servomotor as Joint Actuator in a Tripod Gait

The plot of the instantaneous power drawn by the micro servomotor with respect to time is similar to the current plot in Figure 15, with the peak power drawn by the motor being approximately $1.25 \mathrm{~W}$. Replacing the micro servomotors with high torque metal-geared servomotor yielded greater current spikes of $650 \mathrm{~mA}$, as shown in Figure 16, which in turn produces a power plot composed of power peaks of $3.38 \mathrm{w}$, which is almost three times the peak power consumed by the micro servomotor, as shown in Figure 18. This shows that our novel hexapod design concept puts less current and power strain on a robot's power supply than conventional hexapod robot designs of similar link parameters. 


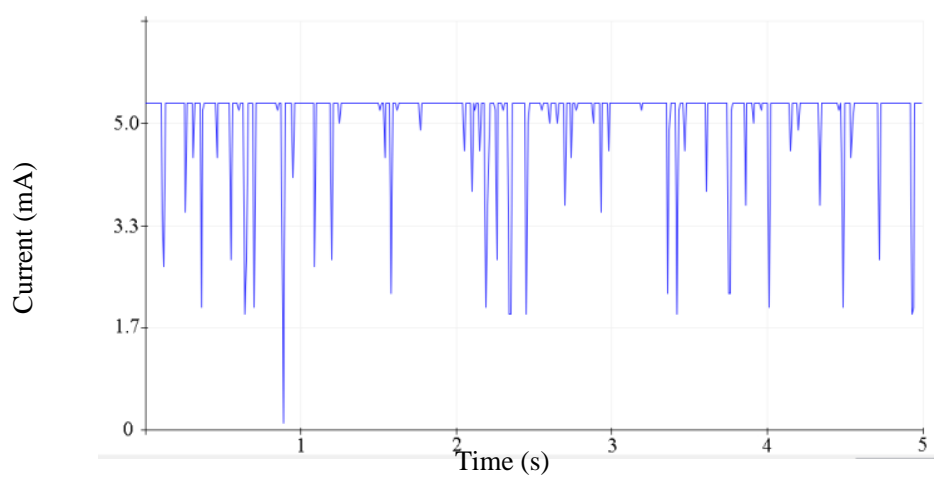

Figure 15. Current Drawn by a Frame 1 Micro Servomotor Joint Actuator.

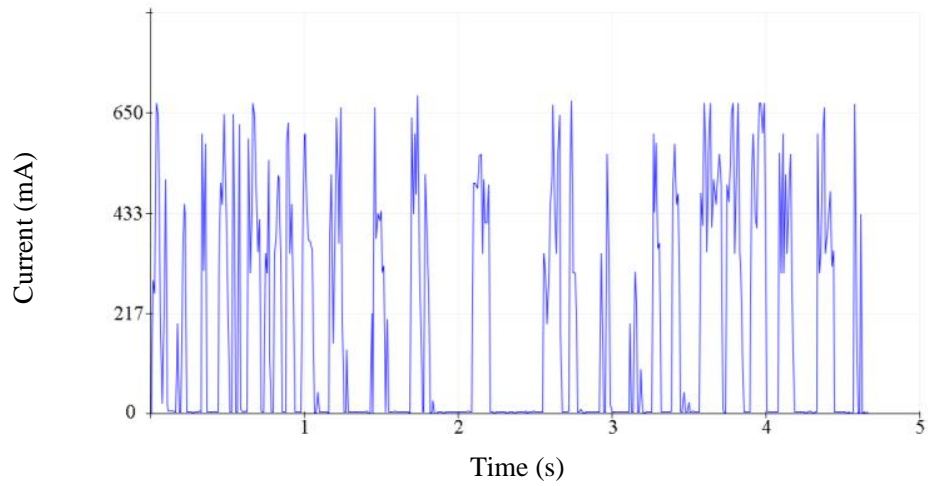

Figure 16. Current Drawn by a Frame 1 MG996r Servomotor Joint Actuator.

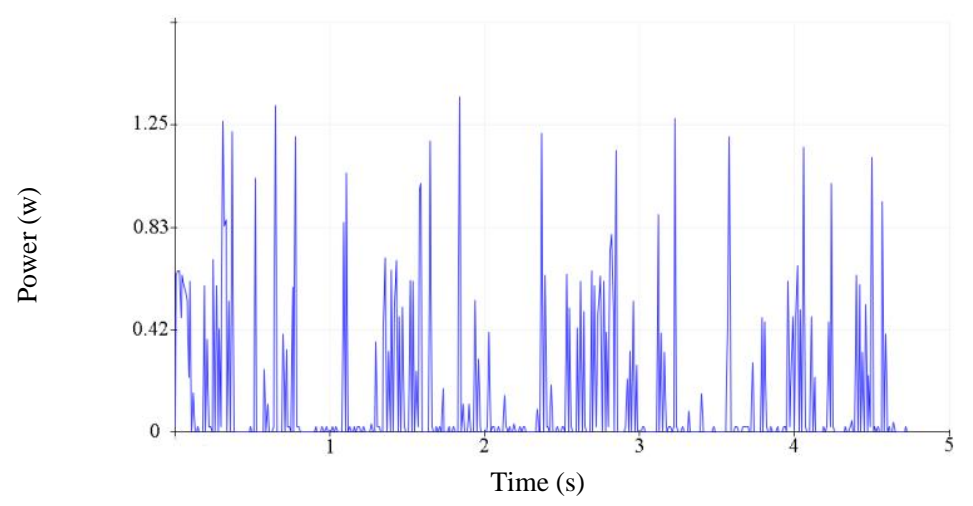

Figure 17. Power Drawn by a Frame 1 Micro Servomotor Joint Actuator.

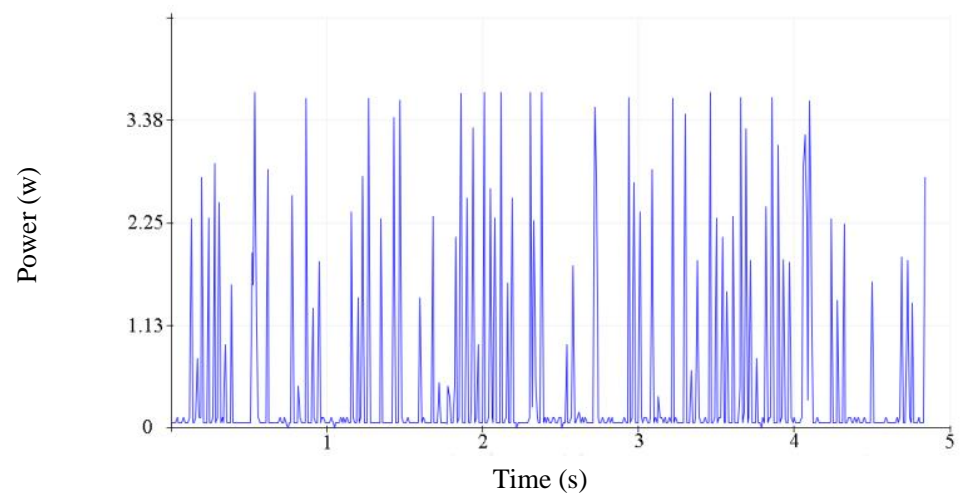

Figure 18. Power Drawn by a Frame 1 MG996r Servomotor Joint Actuator. 


\subsection{Orientation Analysis}

We also used chassis vibrations and orientation changes as performance metrics to compare our novel design to conventional hexapod design. The MPU-6050 Inertia Measurement Unit (IMU), which is composed of an accelerometer and a gyroscope, was used to obtain the orientation changes in the hexapod robot using both our novel design and the conventional one. The accelerometer readings were obtained in terms of the acceleration due to gravity $(\mathrm{g})$, which is approximately $9.8 \mathrm{~m} / \mathrm{s} 2$, as the robot executes a tripod walking sequence across a horizontal platform with a hard and non-textured surface. Figure 19 shows the roll angle readings of the prototype robot while executing a tripod walking sequence using conventional designs, as evaluated from the accelerometer readings, and Figure 20 shows the pitch angle readings. Figures 21 and 22 show the roll and pitch angles of the prototype hexapod when it is configured with the weight-bearing wheel. From these figures it is seen that there are greater oscillations in the pitch and roll angles of the prototype robot when it was configured using conventional designs than when the weight-bearing is attached. The roll angle in the conventional configuration oscillates between $+18^{\circ}$ and $-8^{\circ}$, and the pitch angle has less pronounced orientation swings, with spans of $10^{\circ}$, but it is saturated with more vibrations. Conversely, the roll and pitch angles with the weightbearing wheel attached had significantly smaller swings in orientation with angle readings being between $0^{\circ}$ and $5^{\circ}$.

Similarly, Figures 23 and 24 show the roll and pitch angular velocity of the prototype robot in the conventional hexapod configuration, which was obtained from the gyroscope embedded in the IMU, where Figures 25 and 26 show the roll and pitch angular velocity with the wheel attached. As shown in these figures the chassis of the prototype robot experienced greater angular velocities in the roll and pitch directions when it was configured using conventional designs than when the wheel was attached. Thus, the accelerometer and gyroscope data show that our novel design is more balanced while executing walking sequences across horizontal platforms than conventional hexapod robot designs.

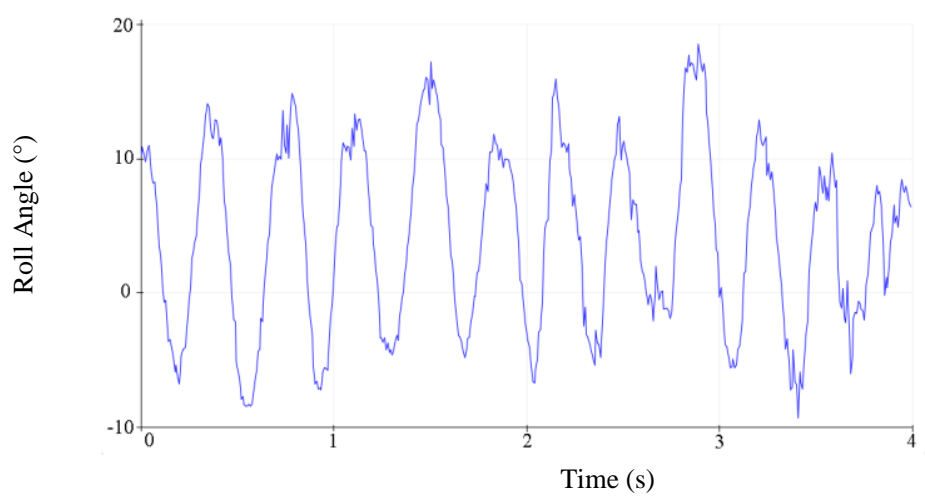

Figure 19. Conventional Hexapod Roll Angle While Executing a Tripod Walking Sequence

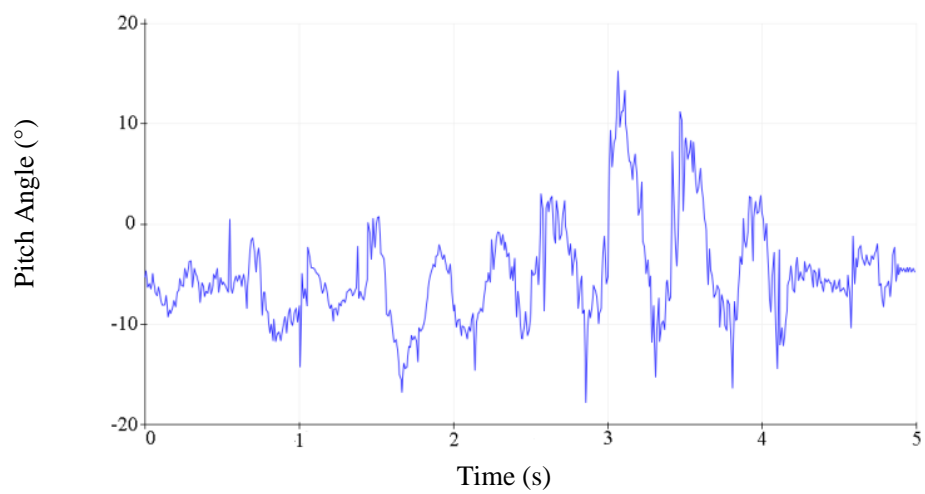

Figure 20. Conventional Hexapod Pitch Angle While Executing a Tripod Walking Sequence 


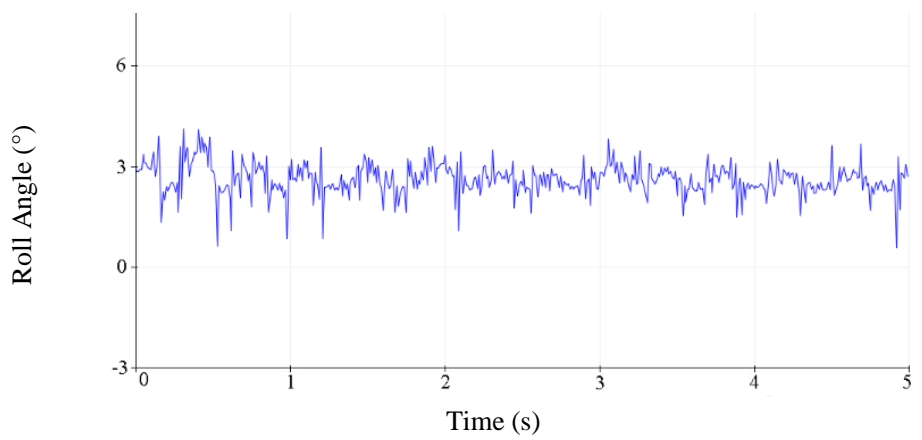

Figure 21. Novel Hexapod Roll Angle While Executing a Tripod Walking Sequence

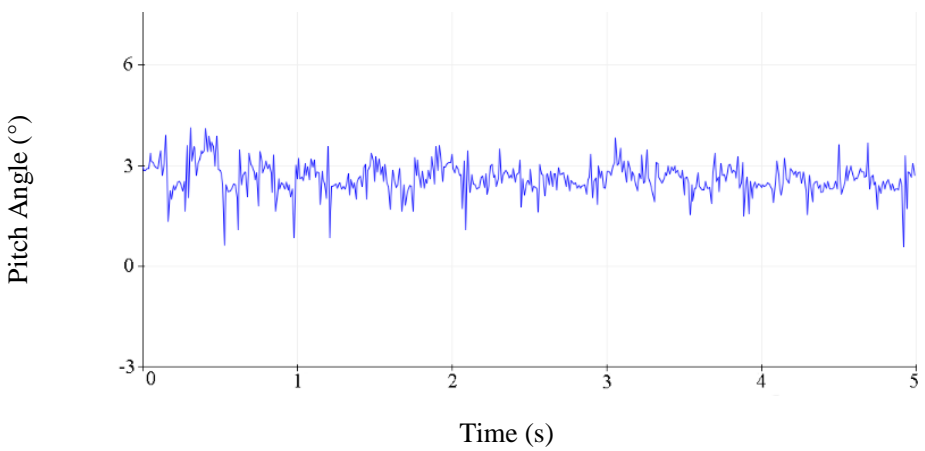

Figure 22. Novel Hexapod Pitch Angle While Executing a Tripod Walking Sequence

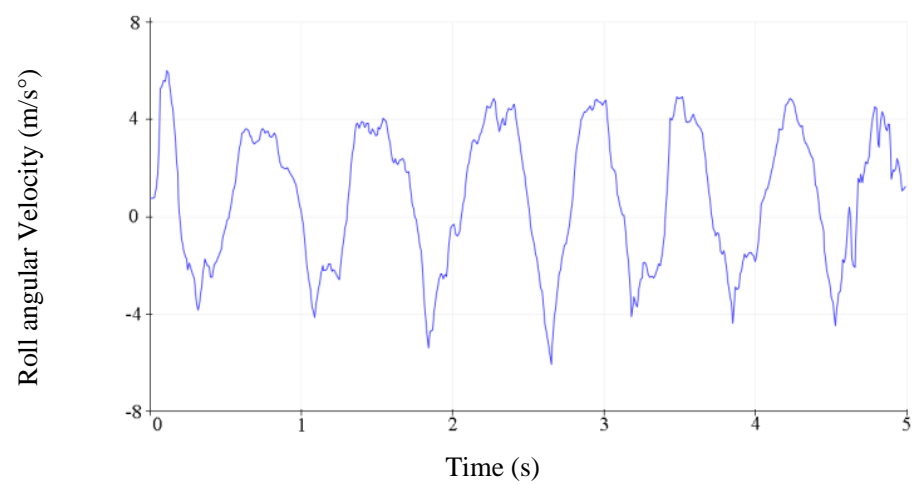

Figure 23. Conventional Hexapod Roll Angular Velocity While Executing a Tripod Walking Sequence 


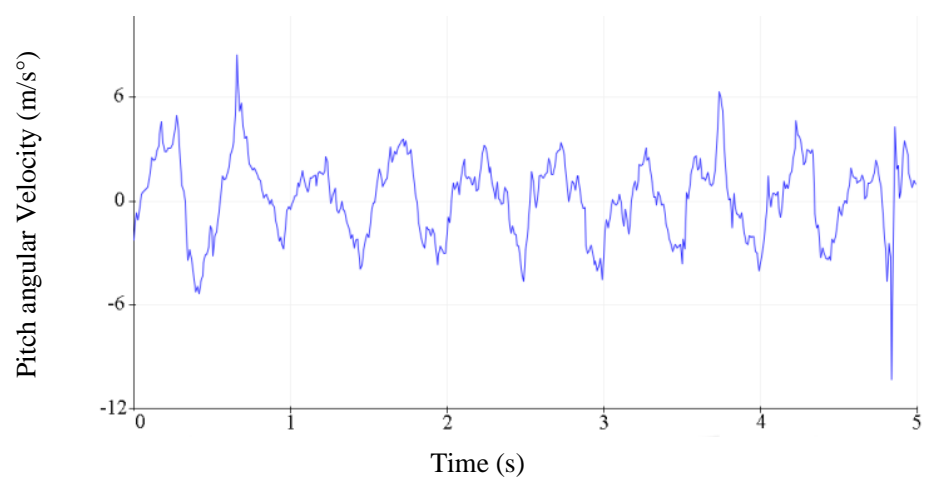

Figure 24. Conventional Hexapod Pitch Angular Velocity While Executing a Tripod Walking Sequence

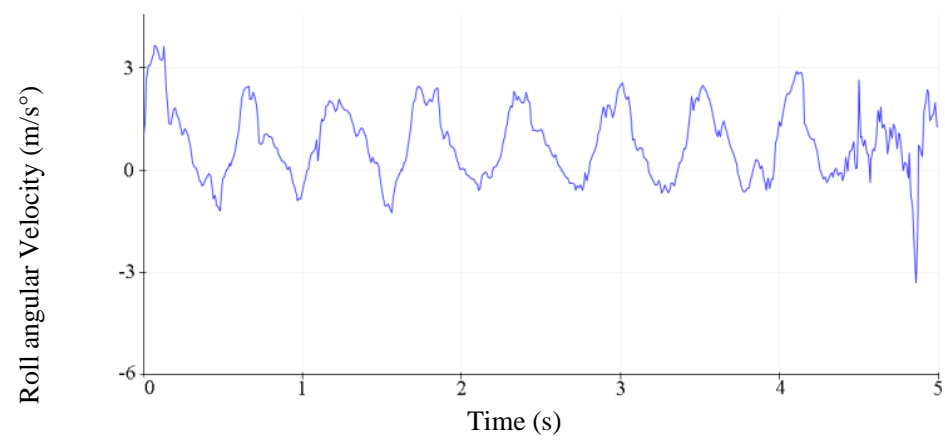

Figure 25. Novel Hexapod Roll Angular Velocity While Executing a Tripod Walking Sequence

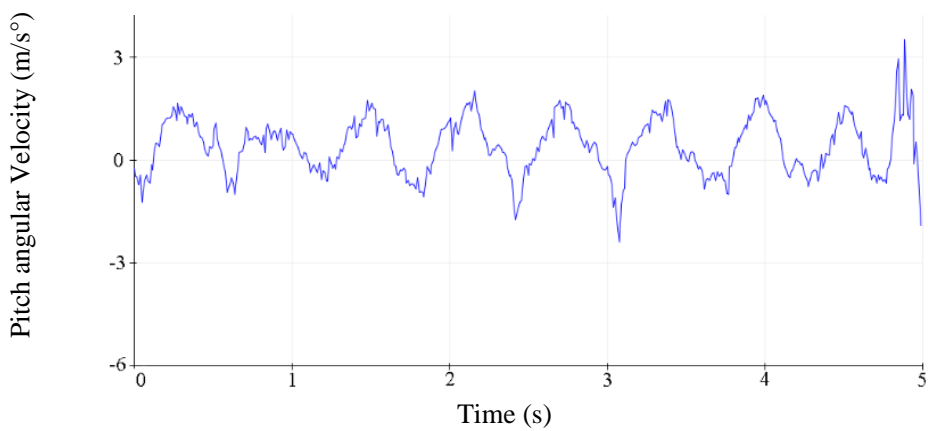

Figure 26. Novel Hexapod Pitch Angular Velocity While Executing a Tripod Walking Sequence

\section{Conclusion and Future Works}

The developed novel prototype hexapod robot design is capable of performing all walking gaits that conventional hexapod robots could perform with less than half the required power consumption. The most unique ability of conventional hexapod robots is to raise their main chassis to allow obstacles to pass underneath 
them. The novel hexapod allows its wheel to roll over small obstacles while the robot's limbs reposition their end-effectors to compensate for the increased height off the ground. Though the novel design shows satisfactory performance across horizontal platforms with smooth or relatively irregular surfaces, it does not include the ability to climb on vertical platforms, over large object or through flights of stairs, which are exclusive abilities of conventional hexapods with high torque joint actuators. Thus, the performance tests and analysis performed on our prototype hexapod robot proves that our novel hexapod design, which involves the incorporation of a rotary encoder-embedded weight-bearing wheel, significantly relaxes the stringent requirements in the choice of joint actuators for hexapod robots while providing a low cost and complexity medium for linear distance measurement, which are the limitation that plaque conventional hexapod designs. More research can be done on the unique applications that suit the deployment our novel hexapod designs.

\section{References}

Asbeck, A. T., Provancher, K. S. \& Lanzet, W. R., (2005). Scaling hard vertical surfaces with compliant microspine arrays.. s.1., s.n., pp. 1165-1179.

Autumn, K. et al., (2005). Kellar Autumn, Martin Buehler, Mark Cutkosky, Ronald Fearing, Robert J. Full, Daniel Goldman, Richard Groff, William ProRobotics in scansorial environments. Florida, s.n.

Autumn, K. et al., 2006. Dynamics of geckos running vertically. Journal of Experimental Biology, Volume 209, pp. 260-272.

Bailey, S. A., Cham, J. G., Cutkosky, M. R. \& Full, R. J., (1999). Biomimetic mechanisms via shape deposition manufacturing.. s.l., s.n., pp. 621-649.

Goldman, D. I., Chen, T. S., Dudek , D. M. \& Full, R. J., (2006). Dynamics of rapid vertical climbing in cockroaches reveals a template. The Journal of Experimental Biology, Volume 209, pp. 2990-3000.

Konstantinos , K., Ioannis, P. \& Herbert, T. G., (2017). A Navigation and Control Strategy for Miniature Legged Robots. IEEE Transactions on Robotics, 33(1), pp. 214-219.

Matt, H., Jaret , M., Matt, F. \& Chris, M., (2010). Development of the Tri-Athlete Lunar Vehicle Prototype.. Proceedings of the 40th Aerospace Mechanisms Symposium, 40(1), pp. 317-326.

Neal, S. \& Alonzo, K., (2016). High Fidelity Yet Fast Dynamic Models of Wheeled Mobile Robots.. IEEE Transactions on Robotics, 32(3), pp. 614-625.

Oriol, B., Montserrat, M. \& Lluis, R., (2016). Planning Wrench-Feasible Motions for Cable-Driven Hexapods. IEEE Transactions on Robotics, 32(2), pp. 442-451.

Perrin , N. et al., (2016). Continuous Legged Locomotion Planning. IEEE Transactions on Robotics , 33(1), pp. 234-239.

Pope , M. T. et al., (2016). A Multimodal Robot for Perching and Climbing on Vertical Outdoor Surfaces. IEEE Transactions on Robotics, 33(1), pp. 38-48.

Spenko, M. et al., (2006). Foot design and integration for bioinspired climbing robots. Florida, s.n.

Travis, B. L. \& James, S. P., 2016. Reaction Wheel Actuation for Improving Planar Biped Walking Efficiency.. IEEE Transactions on Robotics, 32(5), pp. 1290-1297., 32(5), pp. 1290-1297.

Wensing, P. M. et al., (2017). Proprioceptive Actuator Design in the MIT Cheetah: Impact Mitigation and HighBandwidth Physical Interaction for Dynamic Legged Robots. IEEE Transactions on Robotics, 33(3), pp. 509-522. 